\title{
Putative carboxylesterase gene identification and their expression patterns in Hyphantria cunea (Drury)
}

Jia Ye ${ }^{\text {Equal first author, } 1}$, Dingze Mang ${ }^{\text {Equal first author, } 2}$, Ke Kang ${ }^{1,3}$, Cheng Chen ${ }^{1}$, Xiaoqing Zhang ${ }^{1}$, Yanping Tang ${ }^{1}$, Endang R. Purba ${ }^{4}$, Liwen Song ${ }^{5}$, Qing-He Zhang ${ }^{6}$, Longwa Zhang ${ }^{\text {Corresp. } 1}$

${ }^{1}$ Anhui Provincial Key Laboratory of Microbial Control, Engineering Research Center of Fungal Biotechnology, Ministry of Education, School of Forestry \& Landscape Architecture, Anhui Agricultural University, Hefei, China

2 Graduate School of Bio-Applications and Systems Engineering, Tokyo University of Agriculture and Technology, Tyoko, Japan

3 Anhui Forestry Bureau, Hefei, China

4 Structural Cellular Biology Unit,, Okinawa Institute of Science and Technology Graduate University, Okinawa, Japan

5 Jilin Provincial Academy of Forestry Sciences, Changchun, China

${ }^{6}$ Sterling International, Inc., Spokane, USA

Corresponding Author: Longwa Zhang

Email address: zhanglw@ahau.edu.cn

The olfactory system of insects is important for behavioral activities as it recognizes internal and external volatile stimuli in the environment. Insect odorant degrading enzymes (ODEs), including antennal-specific carboxylesterases (CXEs), are known to degrade redundant odorant molecules or to hydrolyze important olfactory sex pheromone components and plant volatiles. Compared to many well-studied Type-I sex pheromoneproducing lepidopteran species, the molecular mechanisms of the olfactory system of Type-II sex pheromone-producing Hyphantria cunea (Drury) remain poorly understood. In the current study, we first identified a total of ten CXE genes based on our previous $H$. cunea antennal transcriptomic data. We constructed a phylogenetic tree to evaluate the relationship of HcunCXEs with other insects' CXEs, and used quantitative PCR to investigate the gene expression of $H$. cunea CXEs (HcunCXEs). Our results indicate that HcunCXEs are highly expressed in antennae, legs and wings, suggesting a potential function in degrading sex pheromone components, host plant volatiles, and other xenobiotics. This study not only provides a theoretical basis for subsequent olfactory mechanism studies on $\mathrm{H}$. cunea, but also offers some new insights into functions and evolutionary characteristics of CXEs in lepidopteran insects. From a practical point of view, these HcunCXEs might represent meaningful targets for developing behavioral interference control strategies against $H$. cunea. 
1 Putative carboxylesterase gene identification and their

2 expression patterns in Hyphantria cunea (Drury)

$3 \mathrm{Jia} \mathrm{Ye}^{1 \dagger}$, Dingze Mang ${ }^{2 \dagger}$, Ke Kang ${ }^{1,3}$, Cheng Chen ${ }^{1}$, Xiaoqing Zhang ${ }^{1}$, Yanping Tang ${ }^{1}$,

4 Endang R. Purba ${ }^{4}$, Liwen Song ${ }^{5}$, Qing-He Zhang ${ }^{6}$, Longwa Zhang ${ }^{1 *}$

$5 \quad{ }^{1}$ Anhui Provincial Key Laboratory of Microbial Control, Engineering Research Center of

6 Fungal Biotechnology, Ministry of Education, School of Forestry \& Landscape

7 Architecture, Anhui Agricultural University, Hefei, 230036, China

$8{ }^{2}$ Graduate School of Bio-Applications and Systems Engineering, Tokyo University of

9 Agriculture and Technology, Koganei 2-24-16, Tokyo 184-8588, Japan

$10{ }^{3}$ Anhui Forestry Bureau, Hefei, 230001, China

$11{ }^{4}$ Structural Cellular Biology Unit, Okinawa Institute of Science and Technology Graduate

12 University, 1919-1 Tancha, Onna-son, Okinawa, 904-0495, Japan

135 Jilin Provincial Academy of Forestry Sciences, Changchun, 130031, China

$14{ }^{6}$ Sterling International, Inc., Spokane, WA, 99216-1616, USA

15 t: These authors contributed equally to this work.

16 Corresponding Author:

17 Longwa Zhang

18 School of Forestry \& Landscape Architecture, Anhui Agricultural University, No. 130,

19 Changjiang West Road, Hefei 230036, P. R. China.

20 E-mail: zhanglw@ahau.edu.cn 


\section{Abstract}

22 The olfactory system of insects is important for behavioral activities as it recognizes

23 internal and external volatile stimuli in the environment. Insect odorant degrading

24 enzymes (ODEs), including antennal-specific carboxylesterases (CXEs), are known to

25 degrade redundant odorant molecules or to hydrolyze important olfactory sex

26 pheromone components and plant volatiles. Compared to many well-studied Type-I sex

27 pheromone-producing lepidopteran species, the molecular mechanisms of the olfactory

28 system of Type-Il sex pheromone-producing Hyphantria cunea (Drury) remain poorly

29 understood. In the current study, we first identified a total of ten CXE genes based on our

30 previous $H$. cunea antennal transcriptomic data. We constructed a phylogenetic tree to

31 evaluate the relationship of HcunCXEs with other insects' CXEs, and used quantitative

32 PCR to investigate the gene expression of $H$. cunea CXEs (HcunCXEs). Our results

33 indicate that HcunCXEs are highly expressed in antennae, legs and wings, suggesting a

34 potential function in degrading sex pheromone components, host plant volatiles, and

35 other xenobiotics. This study not only provides a theoretical basis for subsequent

36 olfactory mechanism studies on $H$. cunea, but also offers some new insights into

37 functions and evolutionary characteristics of CXEs in lepidopteran insects. From a

38 practical point of view, these HcunCXEs might represent meaningful targets for

39 developing behavioral interference control strategies against $H$. cunea. 


\section{Introduction}

43 A complete insect olfactory process requires the participation and cooperation of various

44 olfaction-related proteins (Scott et al., 2001; Vogt, 2003; Leal, 2013). During the process,

45 external liposoluble odor molecules first pass through the polar pores on the sensillum

46 surface, then enter the lymph under the integument where they further combine with

47 odorant binding proteins (OBPs) before being transferred to the dendritic membrane of

48 olfactory receptor neurons (ORNs) (Tegoni, Campanacci \& Cambillau, 2004; Leal, 2013;

49 Pelosi et al., 2018). The molecule-bound odorant receptors (ORs) then convert the chemical

50 signals into electrical signals that are transmitted to the central nervous system through

51 axons of the ORNs (Song et al., 2008). This whole process guides insects to make

52 relevant physiological responses and behavioral decisions. Once the signal transmission

53 is completed, redundant odorant molecules need to be degraded or inactivated by

54 odorant degrading enzymes (ODEs) in the antennal sensilla; otherwise, the odorant

55 receptors will remain in a stimulated state, which may lead to poor spatio-temporal

56 resolution of the odor signal, and pose fatal hazards to the insects (Vogt \& Riddiford,

57 1981; Steinbrecht, 1998; Durand et al., 2010b; Leal, 2013). ODEs degrade redundant

58 odorant molecules in the lymph of antennal sensilla and within the cells (He et al., 2014a).

59 Traditionally, ODEs can be divided into five categories based on the structural difference

60 of various target substances: carboxylesterase (CXE), cytochrome P450 (CYP), alcohol 
61 dehydrogenase (AD), aldehyde oxidase (AOX) and glutathione S-transferase (GST)

62 (Rybczynski, Reagan \& Lerner, 1989; Ishida \& Leal, 2005; Pelletier et al., 2007; Durand

63 et al., 2010a). However, ODEs of different categories have been shown to catalytically

64 interact with odor molecules of the same type and structure. It is currently believed that

65 the different enzyme families of ODEs may work together in degradation and clearing of

66 the same type of odor molecule (Steiner et al., 2019).

67 As primary metabolic enzymes, CXEs are widely distributed among insects, microbes

68 and plants (Guo \& Wong, 2020). The active site contains several conserved serines,

69 which promote the cleavage and formation of ester bonds (Bornscheuer, 2002) and play

70 an important role in the metabolism of heterologous substances, pheromone

71 degradation, neurogenesis, developmental regulation and many other functions ( $Y u$ et

72 al., 2009). In addition to the metabolism and detoxification of endobiotics and xenobiotics,

73 another important role of CXEs is to maintain the sensitivity of ORNs. The CXEs enable

74 rapid degradation of stray odors and prevent vulnerable ORNs from being continuously

75 invaded by harmful volatile xenobiotics ( $L i$ et al., 2013). So far, a large number of genes

76 encoding CXEs have been identified and their functions in insect olfaction have also

77 been investigated in various insects, including Drosophila melanogaster, Mamestra

78 brassicae, Antheraea polyphemus, Sesamia nonagrioides, Popillia japonica, Spodoptera

79 littoralis, Epiphyas postvittana, Agrilus planipennis, S. litura, S. exigua. (Vogt, Riddiford \&

80 Prestwich, 1985; Maïbèche-Coisne et al., 2004; Ishida \& Leal, 2005; Merlin et al., 2007; 
81 Ishida \& Leal 2008; Jordan et al., 2008; Durand et al., 2010b; Mamidala et al., 2013; He

82 et al., 2014a; He et al., 2014b; He et al., 2014c; He et al., 2015; Chertemps et al., 2015).

83 For instance, the A. polyphemus pheromone-degrading enzyme CXE (Apo/PDE) was

84 shown to effectively degrade its sex pheromone acetate component (Maïbèche-Coisne

85 et al., 2004; Ishida \& Leal, 2005). In P. japonica and D. melanogaster, the purified native

86 or recombinant antennal CXEs were found to degrade their sex pheromone constituents

87 (Ishida \& Leal, 2008; Younus et al., 2014). In addition, some of CXEs from S. exigua, S.

88 littoralis and S. litura were also found to degrade both their sex pheromones and plant

89 volatiles, as well as hydrolyze volatile esters released from their natural food sources

90 (Gomi, Inudo \& Yamada, 2003; Durand et al., 2011; Chertemps et al. 2015).

91 The fall webworm, Hyphantria cunea (Drury) (Lepidoptera; Erebidae), native to North

92 America, is a worldwide quarantine pest insect. This moth has now spread to most

93 European countries (except the Nordics), South Korea, North Korea and China, and

94 lately to Central Asia (Itô \& Miyashita, 1968; Gomi, 2007). As an invasive pest, H. cunea

95 was first found in Dandong (Liaoning province, China) and has rapidly spread to Hebei

96 and adjacent provinces in China (Gomi, 2007; Yang et al., 2008; Tang, Su \& Zhang,

97 2012). In 2012, the State Forestry Administration's Forest Pest Inspection and

98 Identification Center identified the first outbreak of $H$. cunea in Sanshan district, Wuhu

99 City, Anhui Province, which was the southernmost known outbreak of $H$. cunea. Its

100 invasion has caused serious damage to local forests, agricultural crops and 
101 landscaping/ornamental trees, resulting in great economic and ecological losses. Thus,

102 effective quarantine programs and environmentally safe pest management solutions are

103 needed to combat this serious invasive pest insect. More importantly, a better

104 understanding of its chemical ecology may facilitate more effective pest management

105 strategies. Previous studies have described four sex pheromone components, including

106 two straight chain aldehydes, (9Z,12Z)-octadecadienal (Z9, Z12-18Ald) and (9Z,12Z,15Z)-

107 octadecatrienal (Z9, Z12, Z15-18Ald), and two epoxides, $(3 Z, 6 Z, 9 S, 10 R)-9,10$-epoxy-

108 3,6-heneicosadiene (Z3, Z6-9S, 10R-epoxy-21Hy) and (3Z,6Z,9S,10R)-9,10-epoxy-

109 1,3,6-heneicosatriene (1, Z3, Z6-9S, 10R-epoxy-21Hy), which are produced by female $H$.

110 cunea (M. et al., 1989). There are two major groups of moth sex pheromones: Type I

111 pheromones and Type II pheromones (M. et al., 1989; Millar, 2000; Ando et al., 2004).

112 Type I pheromones mostly contain $\mathrm{C}_{10}-\mathrm{C}_{18}$ unsaturated hydrocarbons and a terminal

113 functional group ( $>75 \%$ moth species). Type II pheromones lack a terminal functional

114 group and contain $\mathrm{C}_{17}-\mathrm{C}_{23}$ unsaturated hydrocarbons and epoxy derivatives (Millar, 2000,

115 Ando et al., 2004). Compared to many well-studied Type-I sex pheromone-producing

116 moth species, the molecular mechanisms of olfaction in the Type-II sex pheromone-

117 producing $H$. cunea are poorly understood. In the current study, a total of 10 CXE genes

118 were identified based on our previous $H$. cunea antennal transcriptomic data (Zhang et

119 al., 2016). To understand the potential physiological roles of these HcunCXEs, we

120 constructed a phylogenetic tree to evaluate the relationship of HcunCXEs with other 
121 insects' CXEs, and used reverse transcription-quantitative PCR (RT-qPCR) and reverse

122 transcription PCR (RT-PCR) to investigate the expression of these genes. We found that

123 HcunCXEs displayed either antennae- or leg/wing-biased expression. The differential

124 expression pattern of HcunCXEs suggests a potential function in degrading pesticides

125 and/or other xenobiotics.

126

127

128 Materials and Methods

129 Insect rearing and tissue collection

130 H. cunea pupae were collected from a first-generation population at Baimao Town,

131 Jiujiang District, Wuhu City, Anhui province. Insect cages were used for rearing $H$. cunea

132 pupae at $25^{\circ} \mathrm{C}, 70-80 \% \mathrm{RH}$ and $14 \mathrm{~L}: 10 \mathrm{D}$ hour photoperiod. After eclosion, adults were

133 provided with $1 \%$ honey water. In the fourth hour of the second dark period, antennae,

134 thoraxes, abdomens, legs, and wings of virgin males and females were dissected under

135 the microscope and pooled by sex and body part. Male and female pupae and fourth

136 instar larvae were also sampled. Five samples were taken for each body part with the

137 exception of antennae, of which 30 pairs were collected by pulling out from the base of

138 the antennae with tweezers. Dissected body parts or whole-body samples were flash

139 frozen in liquid nitrogen and stored at $-80^{\circ} \mathrm{C}$ until use. 


\section{Gene annotation}

142 The $H$. cunea antennal transcriptome (PRJNA605323) (Zhang et al., 2016) was used as

143 a reference sequence for mapping clean reads for each tested sample. Gene annotation

144 was carried out using $\mathrm{Nr}$ (NCBI non-redundant protein sequences), Nt (NCBI nucleotide),

145 Pfam (Protein family), KOG/COG (Clusters of Orthologous Groups of

146 proteins/enKaryotic Ortholog Groups), Swiss-Prot (A manually annotated and reviewed

147 protein sequence database), KEGG (Kyoto Encyclopedia of Genes and Genomes) and

148 GO (Gene Ontology) databases (Fig. S1-4). Based on the results of gene annotation and

149 BLAST comparison, the candidate genes of HcunCXE were determined and named

150 according to the identification order from the antennal transcriptomic data.

151

152 Homologous search and sequencing analysis of CXE genes in $\boldsymbol{H}$. cunea

153 The $H$. cunea CXE genes were identified according to the BLAST results on NCBI. The

154 Open Reading Frame finder (OFR Finder) (https://www.ncbi.nlm.nih.gov/orffinder/) was

155 used to search for the open reading frame of these CXE genes. An ExPASy tool

156 (http://web.expasy.org/compute_pi/) (Petersen et al., 2011) was used to calculate their

157 theoretical isoelectric points (pl) and molecular weights (MW) of the full-length

158 HcunCXEs gene candidates, and SignalP-5.0

159 (https://services.healthtech.dtu.dk/service.php?SignalP) was used to predict signal

160 peptides of the CXE genes (Petersen et al., 2011). 


\section{Phylogenetic analysis of CXE genes in $\boldsymbol{H}$. cunea}

163 Genes related to the CXEs of H. cunea and other reported insects (Seasamia inferens,

164 Spodoptera littoralis, Spodoptera exigua, Cnaphalocrocis medinalis, Bombyx mori,

165 Drosophila melanogaster, Tribolium castaneum, Mamestra brassicae and Antheraea

166 polyphemus) were subjected to multi-sequence alignment with MAFFT (Wong et al.,

167 2008). Amino acid sequences were automatically aligned by the MAFFT program

168 version 7 (http://mafft. cbrc.jp/alignment/software/algorithms/algorithms.html), using L-

169 INS-i strategy (Katoh \& Standley, 2013). The phylogenetic tree was constructed using

170 MEGA-X (Tamura et al., 2011) and maximum likelihood method (1000 bootstrap

171 repetitions) for systematic evolution analysis. The adopted model was LG-G+I, and all sites

172 were used for Gap/Missing Data Treatment. Lastly, the phylogenetic tree was edited on the

173 website iTOL (https://itol.embl.de/). The genes of insect ODEs required for the

174 phylogenetic tree are shown in Supplementary Table S1.

175

176 RNA extraction and synthesis of the first-strand cDNA

177 The sampled body tissues were ground using a Tissue-Tearor which rapidly

178 homogenized the samples in DEPC-treated sterile water. TRIzol reagent (Invitrogen, USA)

179 was used for extraction and purification of total RNA from each sample according to the

180 manufacturer's instructions. The degradation and contamination of RNA was monitored on 
$1811 \%$ agarose gels, and purity was checked using a NanoPhotometer® spectrophotometer

182 (IMPLEN, CA, USA). First-stranded cDNA templates were synthesized using $1 \mu \mathrm{g}$ of

183 RNA template with the PrimeScript ${ }^{\mathrm{TM}} \mathrm{RT}$ reagent Kit according the manufacturer's

184 instructions (TaKaRa, Japan).

185

186

\section{RT-qPCR and RT-PCR analysis}

187 Expression profiles of the identified $H$. cunea CXE genes in different body parts of adults 188 and two other life stages were analyzed. Tissues included antenna of 30 adults of each

189 sex, legs of 5 adults of each sex, wings of 5 adults of each sex, thoraxes and abdomens

190 of 5 adults of each sex, 5 whole pupae of each sex and 5 larvae (fourth instar).

191 The RT-qPCR and RT-PCR assays were employed for production of multiple copies of

192 DNA. RT-qPCR reaction was conducted in a $25 \mu \mathrm{L}$ reaction mixture system containing $12.5 \mu \mathrm{L}$ of

193 SYBR ${ }^{\circledR}$ Premix Ex Taq II (Tli RNaseH Plus) (TaKaRa, Japan), $1 \mu \mathrm{L}$ of each primer, $2 \mu \mathrm{L}$ of

194 sample cDNA, and $8.5 \mu \mathrm{L}$ of sterilized $\mathrm{H}_{2} \mathrm{O}$.

195 The RT-qPCR cycles were set at $95^{\circ} \mathrm{C}$ for $30 \mathrm{sec}$, followed by 40 cycles at $95^{\circ} \mathrm{C}$ for $5 \mathrm{sec}$,

$19660^{\circ} \mathrm{C}$ for $30 \mathrm{sec}$. Each experiment was carried out in a CFX96 real-time PCR detection

197 instrument (Bio-rad, USA) using 8-strip PCR tubes (Bio-rad, USA). The reaction data were

198 recorded, and the dissolution curves were appended. Both Elongation factor-1 alpha (EF1-a) and

199 glyceraldehyde-3-phosphate dehydrogenase (GAPDH) were used as internal reference. Three 
biological replicates were performed, and the reproducibility confirmation of each RT-qPCR

201 reaction was replicated three times for each sample (Table S2) (Xu et al., 2018).

202 The variability of each gene expression in different body tissues was tested by using the Q-

203 Gene method (Muller et al., 2002; Simon, 2003). The relative expression of mRNA of each gene

204 (mean \pm SD) was analyzed using one-way ANOVA (SPSS22.0 for Windows, IBM, USA),

205 followed by LSD and Duncan's tests at $\alpha=0.05$. GraphPad Prism v5.0 Software (GraphPad

206 Software Inc, CA, USA) was used for graphical plotting/mapping.

207 RT-PCR analysis was performed as follows: $94^{\circ} \mathrm{C}$ for 2 min of initiation, and 29 cycles of

$20894^{\circ} \mathrm{C}$ for $30 \mathrm{sec}, 52^{\circ} \mathrm{C}$ for $30 \mathrm{sec}, 72^{\circ} \mathrm{C}$ for $15 \mathrm{sec}$, and $2 \mathrm{~min}$ at $72^{\circ} \mathrm{C}$ for final extension.

209 Elongation factor-1 alpha (EF1-a) of $H$. cunea was used as an internal reference. In addition,

210 instead of template cDNA, RNase-free water was used as the blank control. The reaction mixture

211 contained $12.5 \mu \mathrm{L}$ of $2 x$ Ex Taq MasterMix (CWBIO, China), $1 \mu \mathrm{L}$ of each primer, $1 \mu \mathrm{L}$ of sample

212 cDNA, and $\mathrm{H}_{2} \mathrm{O}$ to bring the total to $25 \mu \mathrm{L}$. A $10 \mu \mathrm{L}$ aliquot of each reaction product was used for

213 gel electrophoresis. The RT-PCR primer sequences of CXE genes in H. cunea are listed in

214 Supplementary Table S3.

215

216 Results

217 Identification of CXE genes from $\boldsymbol{H}$. cunea

218 Based on a comparative analysis of the $H$. cunea antennal transcriptome using BLASTX

219 databases (Zhang et al., 2016), a total of 10 HcunCXE genes were identified. BLASTX 
220 comparison showed that these 10 HcunCXE genes have high homology with CXE genes

221 of S. inferens. Six HcunCXEs (HcunCXE1, HcunCXE3-5 and HcunCXE7-8) had

222 complete ORFs (Table 1). The molecular weights of these HcunCXEs ranged from 10.52

223 to $62.23 \mathrm{kDa}$ (Table 2). Only HcunCXE7 and HcunCXE9 have predicted signal peptide

224 sites (Table 2).

225

\section{Phylogenetic analysis of $\boldsymbol{H}$. cunea CXEs}

227 To evaluate the relationship of HcunCXEs with other insects' CXEs, a phylogenetic tree

228 was constructed (Fig. 1). The HcunCXEs genes could be divided into two subclasses:

229 extracellular gene subclass (generally secreted enzymes, substrates include hormone

230 and pheromones) and generally intracellular enzymes, dietary metabolism/ detoxification

231 functions (Fig. 1). Three HcunCXEs (HcunCXE1, 7 and 9) were clustered in the

232 generally secreted enzymes subclass. The other 7 HcunCXEs including HcunCXE2-6,

233 HcunCXE8 and HcunCXE10 fell into the intracellular gene subclass. In addition, the

234 clade of intracellular gene subclass formed by HcunCXEs was most closely related to

235 those formed by S. inferens, C. medinalis, S. exigua and S. littoralis CXEs. Sequence

236 alignments showed that the amino acid identities of HcunCXE1 and SinfCXE18,

237 HcunCXE9 and SinfCXE1, HcunCXE7 and SinfCXE13, HcunCXE7 and CmedCXE5

238 were $73.9 \%, 71.3 \%, 74.6 \%$ and $65 \%$, respectively (Fig. S5). These results suggest that

239 the intracellular CXEs in $H$. cunea shared a more recent common ancestor with the 
240 CXEs in S. inferens, C. medinalis, S. exigua and S. littoralis than with the CXEs in other

241 insect species.

242

\section{Tissue distribution of HcunCXEs}

244 We next examined the expression of HcunCXE genes in adult female and male

245 antennae, legs and wings using RT-qPCR with primers specific for each of the 10

246 HcunCXEs genes (Table S2). All HcunCXEs were expressed in the antennae (Fig. 2 and

247 Fig. S6). Among which, three HcunCXEs (HcunCXE4, 5, 8) were highly expressed in the

248 antennae (Fig. S6 C and D). Two HcunCXEs (HcunCXE1 and 3) were female-biased

249 (Fig. 2 A and C) and two HcunCXEs (HcunCXE 9 and 10) were male-biased (Fig. 2 I and

$250 \mathrm{~J})$; although the sex-biased expression is not statistically significant, there is a clear

251 numerical difference between expression level in the sexes. These results indicate that the

252 most abundant CXE genes in the antenna are not extracellular CXEs that likely participate in

253 volatile odorant degradation. The most abundant CXEs are likely involved in primary metabolic

254 activities and it would thus seem logical that their expression is much higher than for the other

255 specialized CXEs in the antenna. The other HcunCXEs, however, were equally expressed

256 in both sexes. Comparing expression across tissues, five HcunCXEs (2, 3, 5, 7 and 8)

257 were highly expressed in the legs and wings (Fig. S6 A and B). Expression of HcunCXE2

258 and HcunCXE7 was higher in the legs or wings than that in the antennae (Fig. 2 B and

$259 \mathrm{G})$. 
260 To investigate whether these HcunCXEs are also expressed in the other body parts

261 or life stages, a RT-PCR experiment was carried out using total RNA samples taken from

$262 H$. cunea adults and other life stages (pupae and larvae). Gel electrophoresis bands

263 were generated from HcunCXE2 products from the adult thoraxes and abdomens (Fig.

264 3). In addition, faint/light bands of HcunCXE7 and HcunCXE8 were detected in both

265 thoraxes and abdomens, as well as the pupae. Interestingly, nine out of 10 HcunCXEs

266 (HcunCXE1-5 and 7-10) were also detected in the larvae, indicating that HcunCXEs are 267 widely expressed in the larval stage.

\section{Discussion}

270 In the current study, 10 putative CXE genes were identified based on our previous $\mathrm{H}$.

271 cunea antennal transcriptomic data (Zhang et al., 2016). All $10 \mathrm{H}$. cunea CXE genes

272 showed high homology to the CXE genes identified in S. inferens (identity $\geq 59 \%$, Fig. 1

273 and Table 1). We speculated that some of these $H$. cunea CXE genes mainly degrade

274 sex pheromone components and host plant volatiles. Unlike many well-studied Type-I

275 sex pheromone-producing lepidopteran insects ( $>75 \%$ moth species), the $H$. cunea sex

276 pheromone is comprised of Type II pheromone components (Ando \& Inomata, 2004). At

277 present, most of the published moth ODEs are from the Type I sex pheromone

278 producing lepidopterans; thus, our study represents the first report of ODE genes from a

279 Type II sex pheromone-producing moth species. $H$. cunea is an extremely polyphagous 
280 species with high fecundity (several hundred eggs/female) and dispersal capacity. H. cunea

281 larvae are generalists, capable of feeding on over 170 species of host plants, including

282 many broad-leaved tree species. To cope with such diverse host plant species, this moth

283 must have developed a series of olfactory receptor neurons to recognize diverse plant

284 volatiles (Zhang et al., 2016). The number $(n=10)$ of CXE genes we identified from $H$.

285 cunea was lower than those of other reported lepidopterans species: 19 in Chilo

286 suppressalis, 35 in the tea geometrid Ectropis obliqua Prout and 76 in B. mori (Yu et al.,

287 2009; Liu et al., 2015; Sun et al., 2017). These results suggest that $H$. cunea does not

288 seem to require more CXEs, since the other ODEs including CYP, AD, AOX and GST

289 are likely involved in odorant degradation in olfactory processes. On the other hand, the

290 difference in number of CXEs in various species might result from differences in sample

291 preparation and sequencing method/depth. In addition, the ecological/evolutionary

292 differences across species may also be a reason. Insects have to adapt to their external

293 environment; different environments lead to the formation of different physiological and

294 behavioral characteristics.

295 The phylogenetic tree analysis showed that HcunCXE1, 7 and 9 belong to the

296 extracellular gene subclass, including the secretory enzymes that likely act on hormones

297 and pheromones (Fig. 1). The remaining 7 CXE genes fell into the intracellular gene

298 subclass (Fig. 1), including intracellular enzymes that mostly play roles in dietary

299 metabolism and detoxification. Chertemps et al. (2012) demonstrated that an 
300 extracellular CXE of $D$. melanogaster, esterase-6 (EST-6), is responsible in or related to

301 the sensory physiological and behavioral responses to its pheromone. A subsequent

302 study found that EST-6 was able to degrade various volatile esters in vitro and function

303 as expected for an ODE which plays a role in the response of the flies to esters

304 (Chertemps et al., 2012). Thus, these $H$. cunea CXE genes (HcunCXE2, 3, 4, 5, 6, 8 and

305 10) may also affect the mating and courtship competitions in $H$. cunea through

306 degradation of some ester kairomones or plant allelochemicals. On the other hand,

307 based on the omnivorous nature of $H$. cunea and its species-specific sex pheromone,

308 these CXE genes may be a unique category of $H$. cunea which degrade odor substances.

309 Antennal-specific or highly expressed esterases belong to the CXE type in the

310 carboxy/cholinesterases (CCEs) family. The first ODE was identified form $A$.

311 polyphemus (ApolSE) as an antenna-specific esterase, with a high ability to degrade the

312 acetate component (E6Z11-16: AC) of its pheromone blend (Vogt \& Riddiford, 1981).

313 Since then, antennal-specific esterases have been cloned from A. polyphemus (Ishida \&

314 Leal, 2002) and Mamestra brassicae Linnaeus (Maïbèche-Coisne et al., 2004). Recent

315 studies show that many insect CXEs are expressed specifically in antennae, and their

316 major functions in olfactory process are to degrade odor molecules. Interestingly, the

317 expression of some HcunCXEs in the legs and wings were found to be higher than those

318 in the antennae (HcunCXE2, 3 and 7). The ten $H$. cunea CXEs genes we identified

319 through the gene expression analysis had a low level of expression in different body 
320 tissues of $H$. cunea adults (Fig. 2 and Fig. S6). However, they were widely expressed in

321 the larvae, which may be related to their extremely broad host plant range that needs

322 more CXEs to degrade large amount of carboxylic acid esters. Our quantitative PCR

323 results (Fig. 2 and Fig. S6) indicated that some HcunCXEs genes were highly expressed

324 in both male and female antennae. HcunCXE1 and HcunCXE9 belong to the same

325 subclass as ApolPDE and MbraCXE (Fig. 1). Previous studies have shown that

326 ApolPDE and MbraCXE function as pheromone degradation enzymes (Maïbèche-

327 Coisne et al., 2004; Ishida and Leal, 2005). These HcunCXEs are likely for degradation

328 of sex-pheromones and/or plant volatiles both from hosts or non-hosts. However, the

329 HcunCXEs genes that were highly expressed in the legs and wings might be related to

330 the degradation of non-volatile substances for contact signals. In addition, a previous

331 study of SexiCXE14 and SexiCXE15 (antennae-enriched carboxylesterase genes in

332 Spodoptera exigua) showed that antenna bias expression plays a role in the degradation

333 of volatile substances and sex pheromones in plants (He et al., 2015). However, the

334 expression of SexiCXE11 was much higher in abdomen and wings, and its activity in

335 hydrolyzing plant volatile substances was stronger than that in degrading ester sex

336 pheromones (He et al., 2019). In the current study, HcunCXE1, 3, 4, 5, 6, 8, 9, and 10

337 showed antenna-biased expression, while the expression of HcunCXE2 and 7 in legs

338 and wings was higher than that in antennae. These results suggested that HcunCXEs 
339 have different functions and may participate in the degradation of host plant volatiles

340 and/or other xenobiotics.

341 CXEs play multiple key roles in the hydrolysis of carboxylic acids esters. CXEs also

342 include some metabolic enzymes that are associated with insecticide resistance ( $L i$,

343 Schuler \& Berenbaum, 2007). Many previous studies in insect CXEs focused on their

344 functions in mediating insecticide resistance (Hemingway \& Karunaratne, 1998; Li,

345 Schuler \& Berenbaum, 2007). In contrast, the mechanisms underlying degradation of

346 plant allelochemicals are still unclear. It has been shown that phenolic glycosides can

347 induce expression of Papilio canadensis CXEs (Lindroth, 1989). Moreover, in Lymantria

348 dispar, the activities of CXEs were positively correlated with the larval survival, indicating

349 that these esterases might be involved in the glycoside metabolism (Lindroth, 1989;

350 Lindroth \& Weisbrod, 1991). In the current study, nine out of 10 HcunCXEs were

351 expressed in the larvae (Fig. 3), indicating that the activities of HcunCXEs may positively

352 correlate with survival of $H$. cunea larvae. Although the gene expression of HcunCXEs in

$353 H$. cunea midgut and some other tissues are still unknown, based on these previous

354 findings, it is reasonable to speculate that HcunCXEs might also play multiple functions

355 in $H$. cunea physiology and metabolism. In addition, a significant increase of CXE activity

356 in the midgut of $S$. litura was observed during uptake of the plant glycoside rutin

357 (Ghumare, Mukherjee \& Sharma, 1989). The CXEs in Sitobion avenae have been

358 suggested to participate in gramine detoxification (Cai et al., 2009). Quercetinrutin and 2- 
359 tridaconone were also found to induce the activities of CXEs in Helicoverpa Armigera

360 (Gao et al., 1998; Mu, Pei \& Gao, 2006). Understanding the specific function of

361 HcunCXEs will require further analyses using in vitro and in vivo methods.

362 Little is known about $H$. cunea olfaction mechanisms at the molecular level,

363 especially concerning how CXEs degrade various semiochemicals in its chemical

364 communication system. Further research is needed to 1) understand the functions of

365 antennal-specific CXEs in $H$. cunea via cloning, expression and purification of these

366 CXEs and enzymatic kinetic analysis; 2) determine the locations/distributions of related

367 CXEs by in-situ hybridization; 3) evaluate the potential correlations between CXE

368 transcription levels and their corresponding electrophysiological and behavioral

369 responses by silencing CXEs via RNA interference (Caplen, 2004), and 4) ultimately

370 discover the mode of action or functionality of CXEs in the olfactory signal conduction

371 (signal inactivation).

372

\section{Conclusions}

374 In summary, we identified 10 CXE genes in $H$. cunea by analyzing its antennal

375 transcriptomic data. These HcunCXEs displayed an antennae-or leg/wing-biased

376 expression. The ubiquitous expression of these HcunCXEs in different tissues and life

377 stages suggest that they have multiple roles, i.e, degradation of odor molecules,

378 metabolism and detoxification of dietary and environmental xenobiotics. Our findings 
379 provide a theoretical basis for further studies on the olfactory mechanism of $H$. cunea

380 and offer some new insights into functions and evolutionary characteristics of CXEs in

381 lepidopteran insects. From a practical point of view, these HcunCXEs might represent

382 meaningful targets for developing behavioral interference control strategies against $H$.

383 cunea.

384

385 Acknowledgments

386 We would like to thank Dr. Jacob D. Wickham (Managing Editor, Integrative Zoology), Dr.

387 Melissa Matthews and Dr. Hong Huat Hoh (OIST Graduate University, Japan) for editing

388 the manuscript, Dr. Tianzi Gu and Zhenchen Wu for helpful suggestions.

389

390 Competing Interests

391 Dr. Qing-He Zhang is an employee of Sterling International, Inc., Spokane, WA, USA.

\section{References}

394 Ando T, Inomata SI, Yamamoto M. 2004. Lepidopteran sex pheromones. Topics in Current Chemistry 239:51-96 DOI 10.1007/b95449.

Bornscheuer UT. 2002. Microbial carboxyl esterases: classification, properties and application in biocatalysis. FEMS Microbiology Reviews 26(1):73-81 DOI 10.1016/S0168-6445(01)00075-4. 
399 Cai QN, Han Y, Cao YZ, Hu Y, Zhao X, Bi JL. 2009. Detoxification of gramine by the 400 cereal aphid Sitobion avenae. Journal of Chemical Ecology 35(3):320-325 DOI 401 10.1007/s10886-009-9603-y.

402 Caplen N. 2004. Gene therapy progress and prospects. Downregulating gene expression: the impact of RNA interference. Gene therapy 11(16):1241-1248 DOI 10.1038/sj.gt.3302324.

Chertemps T, François A, Durand N, Rosell G, Dekker T, Lucas P, Maïbèche-

Coisne M. 2012. A carboxylesterase, Esterase-6, modulates sensory physiological and behavioral response dynamics to pheromone in Drosophila. BMC biology 10(1):56 DOI 10.1186/1741-7007-10-56. JG, and Maibeche M. 2015. An antennal carboxylesterase from Drosophila melanogaster, esterase 6 , is a candidate odorant-degrading enzyme toward food odorants. Frontiers in physiology 6:315 DOI 10.3389/fphys.2015.00315.

Durand N, Carot-Sans G, Bozzolan F, Rosell G, Siaussat D, Debernard S, volatile components by a same odorant-degrading enzyme in the cotton leafworm, Spodoptera littoralis. PLOS ONE 6(12):e29147-688 DOI 10.1371/journal.pone.0029147. 
418 Durand N, Carot-Sans G, Chertemps T, Bozzolan F, Party V, Renou M, Debernard S,

419 Rosell G, Maïbèche-Coisne M. 2010a. Characterization of an antennal carboxylesterase from the pest moth Spodoptera littoralis degrading a host plant

421 odorant. PLOS ONE 5(11):e15026 DOI 10.1371/journal.pone.0015026.

Durand N, Carot-Sans G, Chertemps T, Montagné N, Jacquin-Joly E, Debernard S, Maïbèche-Coisne M. 2010b. A diversity of putative carboxylesterases are expressed in the antennae of the noctuid moth Spodoptera littoralis. Insect

Gao XW, Zhao Y, Wang X, Dong X, Zheng B. 1998. Induction of carboxylesterasein Helicoverpa Armigera by insecticides and plant allelohemicals. Acta Entomologica Sinica 41:5-11 DOI 10.16380/j.kcxb.1998.s1.002.

Ghumare S, Mukherjee S, Sharma R. 1989. Effect of rutin on the neonate sensitivity, dietary utilization and mid-gut carboxylesterase activity of Spodoptera litura (Fabricius) (Lepidoptera: Noctuidae). Proceedings: Animal Sciences 98(6):399-

Gomi T. 2007. Seasonal adaptations of the fall webworm Hyphantria cunea (Drury) 22(6):855-861 DOI 10.1007/s11284-006-0327-y. 
436 Gomi T, Inudo M, Yamada D. 2003. Local divergence in developmental traits within a trivoltine area of Hyphantria cunea Drury (Lepidoptera: Arctiidae). Entomological Science 6:71-75 DOI 10.1046/j.1343-8786.2003.00010.x.

Guo S, Wong SM. 2020. A Conserved Carboxylesterase Inhibits Tobacco mosaic virus (TMV) Accumulation in Nicotiana benthamiana Plants. Viruses 12(2):195 DOI

\section{2} 10.3390/v12020195.

He P, Mang DZ, Wang H, Wang MM, Ma YF, Wang J, Chen GL, Zhang F, and He M. 2019. Molecular characterization and functional analysis of a novel candidate of cuticle carboxylesterase in Spodoptera exigua degradating sex pheromones and plant volatile esters. Pesticide biochemistry and physiology 163:227-234 DOI 10.1016/j.pestbp.2019.11.022.

He P, Li ZQ, Liu CC, Liu SJ, Dong SL. 2014a. Two esterases from the genus Spodoptera degrade sex pheromones and plant volatiles. Genome 57(4):201-208 DOI 10.1139/gen-2014-0041.

He P, Zhang J, Li ZQ, Zhang YN, Yang K, Dong SL, He P. 2014b. Functional characterization of an antennal esterase from the noctuid moth, Spodoptera exigua. Archives of Insect Biochemistry and Physiology 86(2):85-99 DOI 10.1002/arch.21164. 
454 He P, Zhang YN, Yang K, Li ZQ, Dong SL. 2015. An antenna-biased carboxylesterase

455 is specifically active to plant volatiles in Spodoptera exigua. Pesticide

456 Biochemistry Physiology 123:93-100 DOI 10.1016/j.pestbp.2015.03.009.

457 He P, Zhang YN, Li ZQ, Yang K, Zhu JY, Liu SJ, Dong SL. 2014c. An antennae-enriched carboxylesterase from Spodoptera exigua displays degradation activity in both plant volatiles and female sex pheromones. Insect Molecular Biology 23(4):475-486 DOI 10.1111/imb.12095.

461

462

463

464

465

466

467

468

469

470

471

472

473

Hemingway J, Karunaratne S. 1998. Mosquito carboxylesterases: a review of the molecular biology and biochemistry of a major insecticide resistance mechanism. Medical and Veterinary Entomology 12(1):1-12 DOI 10.1046/j.13652915.1998.00082.x.

Ishida Y, Leal WS. 2002. Cloning of putative odorant-degrading enzyme and integumental esterase cDNAs from the wild silkmoth, Antheraea polyphemus. Insect Biochemistry and Molecular Biology 32(12):1775-1780 DOI 10.1016/S0965-1748(02)00136-4.

Ishida Y, Leal WS. 2005. Rapid inactivation of a moth pheromone. Proceedings of the National Academy of Sciences 102(39):14075-14079 DOI 10.1073/pnas.0505340102.

Ishida Y, Leal WS. 2008. Chiral discrimination of the Japanese beetle sex pheromone and a behavioral antagonist by a pheromone-degrading enzyme. Proceedings of 
474

475

476

477

478

479

480

481

482

483

484

485

486

487

488

489

490

491

492

493

the National Academy Sciences 105(26):9076-9080 DOI

10.1073/pnas.0802610105.

Itô Y, Miyashita K. 1968. Biology of Hyphantria cunea Drury (Lepidoptera: arctiidae) in Japan. V. Preliminary life tables and mortality data in urban areas. Population Ecology 10(2):177-209 DOI 10.1007/BF02510872.

Jordan M, Stanley D, Marshall S, De Silva D, Crowhurst R, Gleave A, Greenwood D, Newcomb R. 2008. Expressed sequence tags and proteomics of antennae from the tortricid moth, Epiphyas postvittana. Insect Molecular Biology 17(4):361-373 DOI 10.1111/j.1365-2583.2008.00812.x.

Katoh K, Standley D. 2013. MAFFT multiple sequence alignment software version 7: improvements in performance and usability. Molecular Biology and Evolution 30(4):772-780 DOI 10.1093/molbev/mst010.

Leal WS. 2013. Odorant reception in insects: roles of receptors, binding proteins, and degrading enzymes. Annual Review of Entomology 58:373-391 DOI 10.1146/annurev-ento-120811-153635.

Li X, Schuler MA, Berenbaum MR. 2007. Molecular mechanisms of metabolic resistance to synthetic and natural xenobiotics. Annual Review of Entomology 52:231-253 DOI 10.1146/annurev.ento.51.110104.151104.

Li Y, Farnsworth CA, Coppin CW, Teese MG, Liu JW, Scott C, Zhang X, Russell RJ, Oakeshott JG. 2013. Organophosphate and pyrethroid hydrolase activities of 
mutant esterases from the cotton bollworm Helicoverpa armigera. PLOS ONE 8(10) DOI 10.1371/journal.pone.0077685.

Lindroth RL. 1989. Host plant alteration of detoxication activity in Papilio glaucus. Entomologia Expermentalis et Applicata 50(1):29-35 DOI 10.1007/BF00190125.

Lindroth RL, Weisbrod AV. 1991. Genetic variation in response of the gypsy moth to aspen phenolic glycosides. Biochemical Systematics and Ecology 19(2):97-103 DOI 10.1016/0305-1978(91)90031-T.

Liu S, Gong ZJ, Rao XJ, Li MY, Li SG. 2015. Identification of putative carboxylesterase and glutathione S-transferase genes from the antennae of the Chilo suppressalis (Lepidoptera: Pyralidae). Journal of Insect Science 1:1 DOI 10.1093/jisesa/iev082. Joly E. 2004. Putative odorant-degrading esterase cDNA from the moth Mamestra brassicae: cloning and expression patterns in male and female antennae. Chemical Senses 29(5):381-390 DOI 10.1093/chemse/bjh039.

Mamidala P, Wijeratne AJ, Wijeratne S, Poland T, Qazi SS, Doucet D, Cusson M, Beliveau C, Mittapalli O. 2013. Identification of odor-processing genes in the emerald ash borer, Agrilus planipennis. PLOS ONE 8(2):e56555 DOI 10.1371/journal.pone.0056555.

512 Merlin C, Rosell G, Carot-Sans G, François MC, Bozzolan F, Pelletier J, Jacquin-Joly E, Guerrero A, Maïbèche-Coisne M. 2007. Antennal esterase 
cDNAs from two pest moths, Spodoptera littoralis and Sesamia nonagrioides, potentially involved in odourant degradation. Insect Molecular Biology 16(1):73-81 DOI 10.1111/j.1365-2583.2006.00702.x.

517 Millar JG. 2000. Polyene hydrocarbons and epoxides: A second major class of lepidopteran sex attractant pheromones. Annual Review of Entomology 45(1):575-604 DOI 10.1146/annurev.ento.45.1.575.

Mu SF, Pei L, Gao XW. 2006. Effects of quercetin on specific activity of carboxylesteras and glutathione S-transferase in Bemisia tabaci. Journal of Applied Entomology 43(004):491-495 DOI 10.3969/j.issn.0452-8255.2006.04.014.

523 Muller P, Janovjak H, Miserez A, Dobbie Z. 2002. Processing of gene expression data generated by quantitative real-time RT PCR. Biotechniques 32(6):1372-1374 DOI 10.1016/S1389-0344(02)00008-4.

Pelletier J, Bozzolan F, Solvar M, François MC, Jacquin-Joly E, Maïbèche-Coisne M. 2007. Identification of candidate aldehyde oxidases from the silkworm Bombyx mori potentially involved in antennal pheromone degradation. Gene 404(1-2):3140 DOI 10.1016/j.gene.2007.08.022.

Pelosi P, lovinella I, Zhu J, Wang G, Dani FR. 2018. Beyond chemoreception: diverse tasks of soluble olfactory proteins in insects. Biological Reviews 93(1):184-200 DOI $\underline{10.1111 / b r v .12339}$. 
533 Petersen TN, Brunak S, Heijne G, Nielsen H. 2011. SignalP 4.0: discriminating signal peptides from transmembrane regions. Nature Methods 8(10):785 DOI 10.1038/nmeth.1701.

536 Rybczynski R, Reagan J, Lerner MR. 1989. A pheromone-degrading aldehyde oxidase in the antennae of the moth Manduca sexta. Journal of Neuroscience 9(4):13411353 DOI 10.1523/JNEUROSCI.09-04-01341.1989.

Scott K, Brady Jr B, Cravchik B, Morozov P, Rzhetsky A, Zuker C, Axel R. 2001. A chemosensory gene family encoding candidate gustatory and olfactory receptors in Drosophila. Cell 104(5):661-673 DOI 10.1016/S0092-8674(01)00263-X.

Simon P. 2003. Q-Gene: processing quantitative real-time RT-PCR data. Bioinformatics 19(11):1439-1440 DOI 10.1093/bioinformatics/btg157.

Song HG, Young Kwon J, Soo Han H, Bae YC, Moon C. 2008. First contact to odors: our current knowledge about odorant receptor. Sensors 8(10):6303-6320 DOI 10.3390/s8106303.

Steinbrecht RA. 1998. Odorant-binding proteins: expression and function. Annals of the New York Academy of Sciences 855:323-332 DOI 10.1111/j.17496632.1998.tb10591.x.

Steiner C, Chertemps T, and Mabèche M. 2019. Diversity of Biotransformation Enzymes in Insect Antennae: Possible Roles in Odorant Inactivation and Xenobiotic Processing. Springer 115-145 DOI 10.1007/978-3-030-05165-5_5. 
553 Sun L, Wang Q, Wang Q, Zhang Y, Tang M, Guo H, Fu J, Xiao Q, Zhang Y, Zhang Y.

5542017 . Identification and expression patterns of putative diversified

555 carboxylesterases in the tea geometrid Ectropis obliqua Prout. Frontiers in

$556 \quad$ Physiology 8:1085 DOI 10.3389/fphys.2017.01085.

557 Tamura K, Peterson D, Peterson N, Stecher G, Nei M, Kumar S. 2011. MEGA5:

558

559

560 molecular evolutionary genetics analysis using maximum likelihood, evolutionary distance, and maximum parsimony methods. Molecular Biology and Evolution

Tang R, Su M, Zhang Z. 2012. Electroantennogram responses of an invasive species fall webworm (Hyphantria cunea) to host volatile compounds. Chinese Science Bulletin 57(35):4560-4568 DOI 10.1007/s11434-012-5356-z. 28(10):2731-2739 DOI 10.1093/molbev/msr121.

28(10):2731-2739 DOl $10.1093 / \mathrm{mol}$

Tang R, Su M, Zhang Z. 2012. Electroantennogram responses of an invasive species

Tegoni M, Campanacci V, Cambillau C. 2004. Structural aspects of sexual attraction and chemical communication in insects. Trends in Biochemical Sciences 29(5):257-264 DOI 10.1016/j.tibs.2004.03.003. BG. 1989. Identification of (3Z,6Z)-1,3,6-9,10-epoxyheneicosatriene and (3Z,6Z)$1,3,6-9,10$-epoxyeicosatriene in the sex pheromone of hyphantria cunea. Tetrahedron Letters 30(26):3405-3408 DOI 10.1016/S0040-4039(00)99256-6. 
571 Vogt R. 2003. 14-Biochemical diversity of odor detection: OBPs. ODEs and SNMPs.

572 Insect Biochemistry and Molecular Biology 397-451 DOI 10.1016/b978-

573 012107151-6/50016-5.

574 Vogt RG, Riddiford LM. 1981. Pheromone binding and inactivation by moth antennae.

575 Nature 293(5828):161-163 DOI 10.1038/293161a0.

576

577

578

579

580

581

582

583

584

585

586

587

588
Xu LT, Zhang YQ, Zhang SH, Deng JD, Lu M, Zhang LW, and Zhang J. 2018. Comparative analysis of the immune system of an invasive bark beetle, Dendroctonus valens, infected by an entomopathogenic fungus. Developmental \& Comparative Immunology 88:65-69 DOI 10.1016/j.dci.2018.07.002.

Yang Z, Wang X, Wei J, Qu H, Qiao X. 2008. Survey of the native insect natural enemies of Hyphantria cunea (Drury) (Lepidoptera: Arctiidae) in China. Bulletin of Entomological Research 98(3):293-302 DOI 10.1017/S0007485308005609. 
589 Younus F, Chertemps T, Pearce SL, Pandey G, Bozzolan F, Coppin CW, Russell RJ,

590 Maïbèche-Coisne M, Oakeshott JG. 2014. Identification of candidate odorant

591 degrading gene/enzyme systems in the antennal transcriptome of Drosophila

592 melanogaster. Insect Biochemistry and Molecular Biology 53:30-43 DOI

$593 \quad$ 10.1016/j.ibmb.2014.07.003.

594 Yu QY, Lu C, Li WL, Xiang ZH, Zhang Z. 2009. Annotation and expression of

595 carboxylesterases in the silkworm, Bombyx mori. BMC genomics 10(1):553 DOI

$596 \quad \underline{10.1186 / 1471-2164-10-553 .}$

597 Zhang LW, Kang K, Jiang SC, Zhang YN, Wang TT, Zhang J, Sun L, Yang YQ,

598 Huang CC, Jiang LY. 2016. Analysis of the antennal transcriptome and insights

599 into olfactory genes in Hyphantria cunea (Drury). PLOS ONE 11(10):e0154729

600 DOI 10.1371/journal.pone.0164729.

601

602

603 Figure legends

604 Figure 1 Molecular phylogeny comparing HcunCXEs with CXEs from other insect

605 species. 10 CEXs (HcunCXE1-10) from H. cunea (Hcun) and CXEs from S. exigua

606 (Sexi), C. medinalis (Cmed), B. mori (Bmor), D. melanogaster (Dmel), T. castaneum

607 (Tcas), S. inferens (Sinf), S. littoralis (Slit) were used to construct the phylogenetic tree.

608 The phylogenetic tree was aligned by MAFFT, and constructed by MEGA-X using 
609 maximum likelihood method. The adopted model is $L G-G+I$, and the model value is 610 shown in table S4 of additional materials. 1000 bootstraps were used to create the tree.

611 A: Extracellular gene subclass (Generally secreted enzymes, substrates include

612 hormone and pheromones); B: Generally intracellular enzymes, dietary metabolism/

613 detoxification functions; C: juvenile hormone esterase (JHE); D: Nerouligins; E: 614 acetylcholinesterases (AChE).

615

616 Figure 2 Relative mRNA expression of HcunCXEs in $\boldsymbol{H}$. cunea tissues. (A-J)

617 HcunCXEs (HcunCXE1, 2, 3, 4, 5, 6, 7, 8, 9 and 10). FA, female antennae; MA, male 618 antennae; L, legs; $\mathrm{W}$, wings. The relative mRNA levels were normalized to those of the

619 EF1-a gene and analyzed using the Q-gene method. All values are shown as the mean \pm 620 SEM. The data were analyzed by the least significant difference (LSD) test after one-way 621 analysis of variance (ANOVA). Different letters indicate significant differences between 622 means $(P<0.05)$.

624 Figure 3 RT-PCR analysis of HcunCXEs gene expression in tissues taken from $\boldsymbol{H}$. 625 cunea adults and other life stages. EF1-a was used as an internal control; NC, 626 negative control with no template in the reaction. 
629 Figure. S1 Homology analysis of $\boldsymbol{H}$. cunea unigenes. (A) E-value distribution. (B)

630 Similarity distribution. (C) Species distribution. All unigenes that had BLASTX

631 annotations within the NCBI nr database with a cutoff $E$-value of $10^{-5}$ were analyzed.

632 The first hit of each sequence was used for analysis.

633

634 Figure. S2 Gene ontology (GO) assignment of $\boldsymbol{H}$. cunea unigenes. The GO 635 classification map was done by uploading the GO ID numbers of genes for their 636 involvement in biological processes, cellular components, and molecular functions.

638 Figure. S3 Clusters of Orthologous Groups (KOG) classification of $\boldsymbol{H}$. cunea. The 639 letters along the $x$-axis represents the name of 26 groups of KOG, and the $y$-axis is the 640 percentage of the number of genes annotated to the group to the total number of genes 641 annotated.

643 Figure. S4 Kyoto Encyclopedia of Genes and Genomes (KEGG) classification of $\boldsymbol{H}$.

644 cunea unigene. The $x$-axis indicates the percentage of annotated genes, and the $y$-axis 645 indicates the KEGG categories. The capital letters against the colored bars indicate five 646 main categories: (A) cellular processes, (B) environmental information processing, (C) 647 genetic information processing, (D) metabolism, and (E) organism systems. 
649 Figure S5. Comparison of the amino acid sequences of HcunCXEs with CXEs

650 proteins from different species. A, HcunCXE1 with SinfCXE18; B, HcunCXE9 and

651 SinfCXE1; C, HcunCXE7 with SinfCXE13 and CmedCXE5. The percentages on the right

652 represent the amino acid identities.

653

654 Figure S6. Relative mRNA expression of HcunCXEs in $\boldsymbol{H}$. cunea tissues. The

655 relative mRNA levels were normalized to those of the EF1-a gene and analyzed using

656 the Q-gene method. All values are shown as the mean \pm SEM. The data were analyzed

657 by the least significant difference (LSD) test after one-way analysis of variance (ANOVA).

658 Different letters indicate significant differences between means $(P<0.05)$. FA, female

659 antennae; MA, male antennae; L, legs; W, wings.

660

661 
Figure 1

Molecular phylogeny comparing HcunCXEs with CXEs from other insect species.

10 CEXs (HcunCXE1-10) from H. cunea (Hcun) and CXEs from S. exigua (Sexi), C. medinalis (Cmed), B. mori (Bmor), D. melanogaster (Dmel), T. castaneum (Tcas), S. inferens (Sinf), S. littoralis (Slit) were used to construct the phylogenetic tree. The phylogenetic tree was aligned by MAFFT, and constructed by MEGA-X using maximum likelihood method. The adopted model is LG-G+I, and the model value is shown in table 4 of additional materials. The Bootstrap value of this tree is 1000 , which is to integrate the branch length tree with the Bootstrap value tree and then beautify it. A: Extracellular gene subclass (Generally secreted enzymes, substrates include hormone and pheromones); B: Generally intracellular enzymes, dietary metabolism/ detoxification functions; C: juvenile hormone esterase (JHE); D: Nerouligins; E: acetylcholinesterases (AChE).

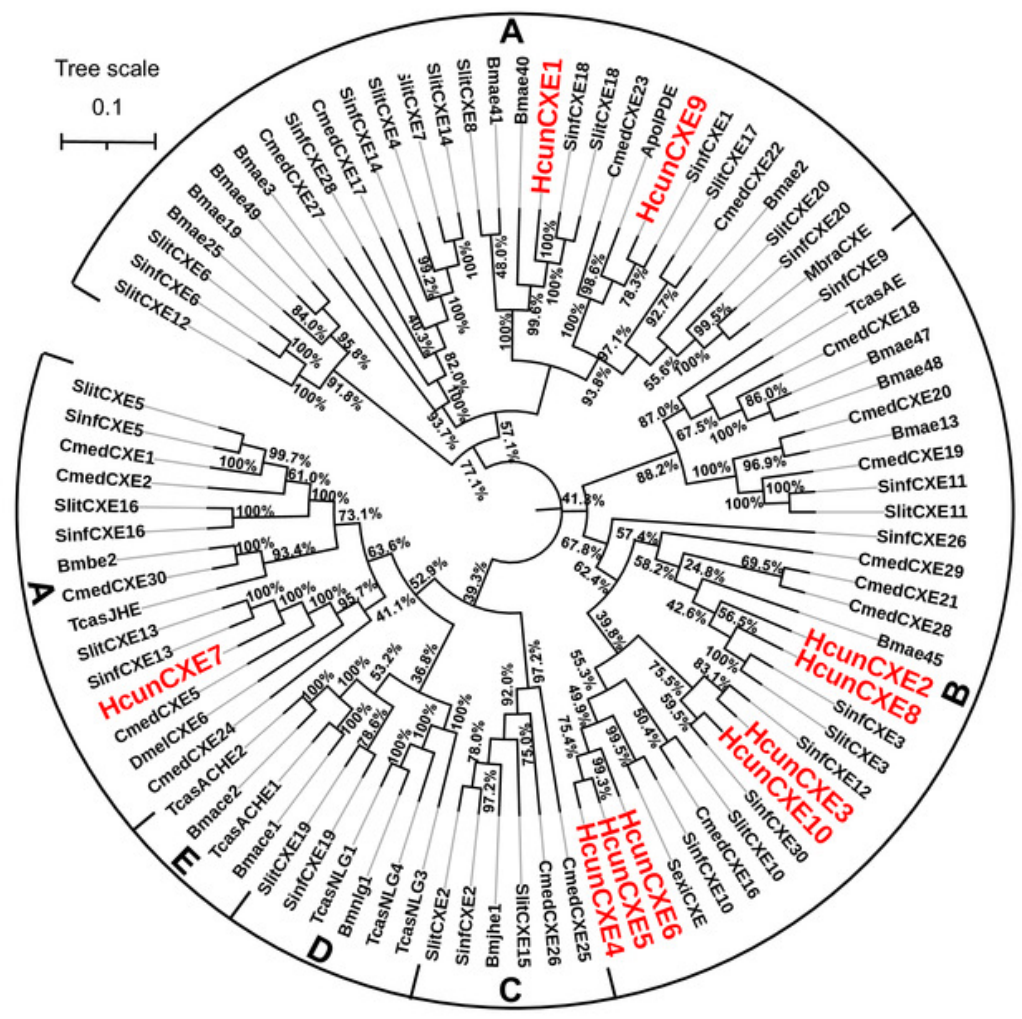




\section{Figure 2}

Relative mRNA expression of HcunCXEs in H. cunea tissues.

(A-J) HcunCXEs (HcunCXE1, 2, 3, 4, 5, 6, 7, 8, 9 and 10). FA, female antennae; MA, male antennae; L, legs; W, wings. The relative mRNA levels were normalized to those of the EF1-a gene and analyzed using the Q-gene method. All values are shown as the mean \pm SEM. The data were analyzed by the least significant difference (LSD) test after one-way analysis of variance (ANOVA). Different letters indicate significant differences between means $(P<$ $0.05)$.

A

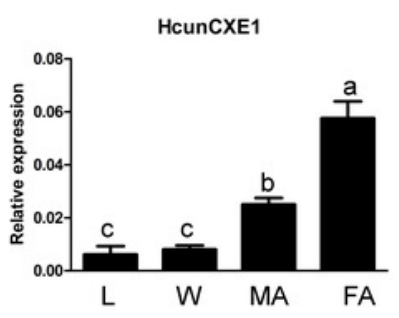

$\mathbf{E}$

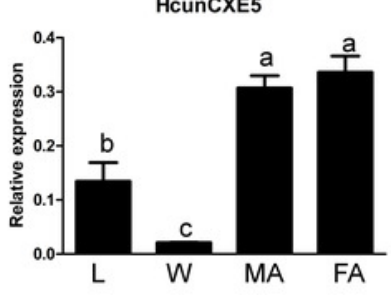

B

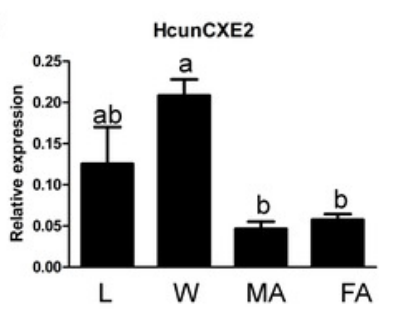

$\mathbf{F}$

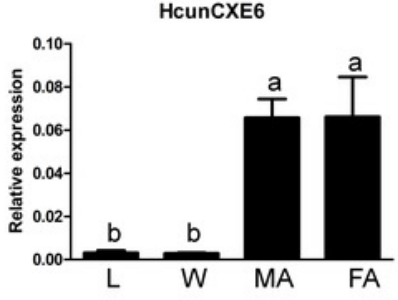

J
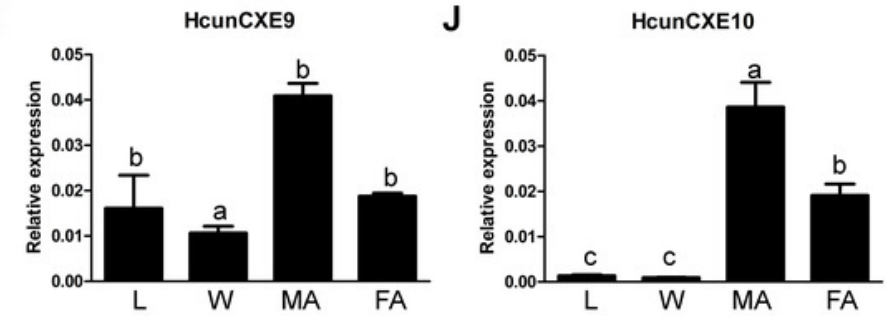

C

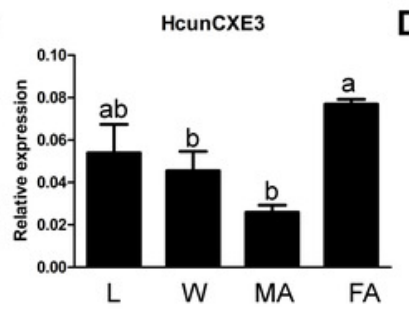

G

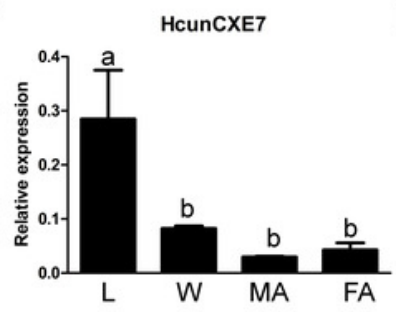

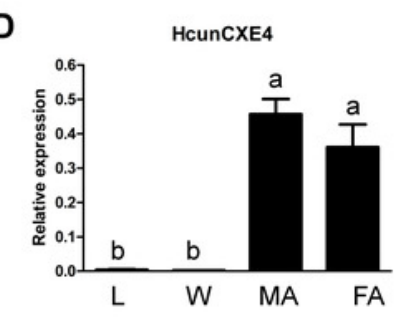

H

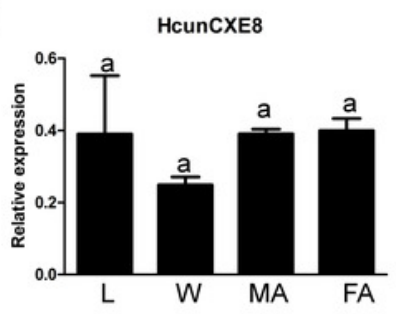




\section{Figure 3}

RT-PCR analysis of HcunCXEs gene expression in tissues taken from $\mathrm{H}$. cunea adults and other life stages.

EF1-a was used as an internal control; NC, negative control with no template in the reaction.

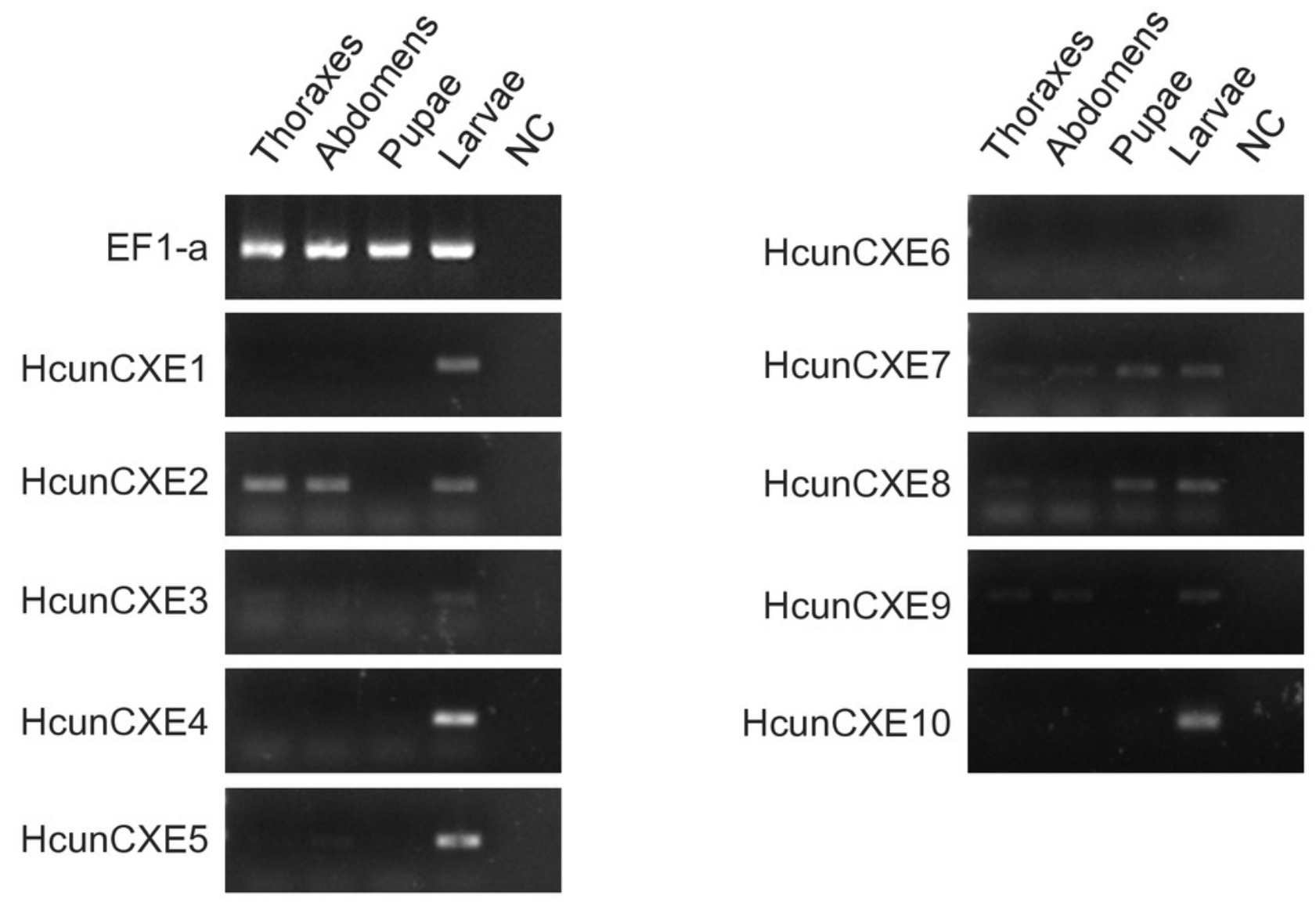




\section{Table $\mathbf{1}$ (on next page)}

Gene name, information of open reading frame and Blastx match of the 10 putative HcunCXEs identified in this study.

Note: ORF, open reading frame. S. inferens, Sesamia inferens. 


\section{Table 1:}

3 Gene name, information of open reading frame and Blastx match of the 10 putative

4 HcunCXEs identified in this study.

\begin{tabular}{lccccccc}
\hline & & & & \multicolumn{4}{c}{ Best Blastx Match } \\
\cline { 5 - 8 } Gene Name & $\begin{array}{c}\text { ORF Length } \\
\text { (bp) }\end{array}$ & $\begin{array}{c}\text { Complete } \\
\text { ORF }\end{array}$ & $\begin{array}{c}\text { FPKM } \\
\text { value }\end{array}$ & Species & Acc.number & $\begin{array}{c}\text { E - } \\
\text { value }\end{array}$ & $\begin{array}{c}\text { Identity } \\
(\%)\end{array}$ \\
\hline HcunCXE1 & 1668 & YES & 4.9 & S. inferens & AII21990.1 & 0.0 & 73 \\
HcunCXE2 & 777 & NO & 3.77 & S. inferens & AII21980.1 & $3 \mathrm{e}-135$ & 73 \\
HcunCXE3 & 375 & YES & 3.26 & S. inferens & AII21980.1 & $2 \mathrm{e}-105$ & 60 \\
HcunCXE4 & 1389 & YES & 61.01 & S. inferens & AII21984.1 & 0.0 & 59 \\
HcunCXE5 & 1593 & YES & 143.14 & S. inferens & AII21984.1 & 0.0 & 62 \\
HcunCXE6 & 1161 & NO & 17.04 & S. inferens & AII21984.1 & $4 \mathrm{e}-174$ & 62 \\
HcunCXE7 & 1677 & YES & 13.18 & S. inferens & AII21987.1 & 0.0 & 75 \\
HcunCXE8 & 1608 & YES & 12.64 & S. inferens & AII21980.1 & 0.0 & 66 \\
HcunCXE9 & 1653 & YES & 6.13 & S.inferens & AII21978.1 & 0.0 & 71 \\
HcunCXE10 & 273 & NO & 21.32 & S. inferens & AII21984.1 & $8 \mathrm{e}-39$ & 64 \\
\hline
\end{tabular}

5 Note: ORF, open reading frame. S. inferens, Sesamia inferens. 


\section{Table 2 (on next page)}

Gene name and characteristics including molecular weight, isoelectric point and signal peptide of the 10 putative HcunCXEs with open reading frames.

Note: SP, signal peptide; pl, isoelectric point; MW, Molecular weight. 
1

2

3

4

5

6

7

8

9

10

11

12

13

14

15

16

17

18

19

20
Table 2:

Gene name and characteristics including molecular weight, isoelectric point and signal peptide of the 10 putative HcunCXEs with open reading frames.

\begin{tabular}{lccc}
\hline Gene Name & MW $(\mathrm{Kda})$ & PI & SP \\
\hline HcunCXE1 & 62.23 & 7.56 & NO \\
HcunCXE2 & 28.44 & 5.67 & NO \\
HcunCXE3 & 13.98 & 4.85 & NO \\
HcunCXE4 & 52.2 & 5.31 & NO \\
HcunCXE5 & 59.52 & 5.41 & NO \\
HcunCXE6 & 43.17 & 5.09 & NO \\
HcunCXE7 & 61.71 & 6.32 & $1-17$ \\
HcunCXE8 & 60.68 & 5.75 & NO \\
HcunCXE9 & 62.18 & 8 & $1-16$ \\
HcunCXE10 & 10.52 & 8.89 & NO \\
\hline
\end{tabular}

Note: SP, signal peptide; $\mathrm{pI}$, isoelectric point; MW, Molecular weight. 